Original article

\title{
Morphological characteristics of interalveolar septum and mandible in BMAL1 gene knockout mice
}

\author{
Ryosuke Koshi $^{1)}$, Kunihito Matsumoto ${ }^{2,3)}$, Yusuke Imanishi ${ }^{2)}$, Takayuki Kawato ${ }^{1,4)}$, Shuichi Sato ${ }^{3,5)}$, Shigeki Shimba ${ }^{6}$, \\ Yoshinori Arai ${ }^{2,3)}$, and Kazuya Honda ${ }^{2,3)}$
}

${ }^{1)}$ Department of Oral Health Sciences, Nihon University School of Dentistry, Tokyo, Japan

${ }^{2}$ Department of Oral and Maxillofacial Radiology, Nihon University School of Dentistry, Tokyo, Japan

${ }^{3)}$ Division of Advanced Dental Treatment, Dental Research Center, Nihon University School of Dentistry, Tokyo, Japan

4) Division of Functional Morphology, Dental Research Center, Nihon University School of Dentistry, Tokyo, Japan

5) Department of Periodontology, Nihon University School of Dentistry, Tokyo, Japan

ब) Department of Health Science, School of Pharmacy, Nihon University, Funabashi, Japan

\begin{abstract}
Purpose: Circadian rhythm is associated with the pathogenesis of systemic disease and bone mineral metabolism. This study aimed to radiographically evaluate morphological characteristics of the interalveolar septum in circadian rhythm deficient animals.

Methods: Heads of 10 brain and muscle arnt-like protein-1 (BMAL1)knockout $(\mathrm{KO})$ mice and 10 wild-type mice sacrificed at 36 weeks were imaged using micro-computed tomography. The mean depth from the cementoenamel junction to the alveolar ridge (virtual bone sounding: VBS) of the interalveolar septum between the first and second molars, and the bone mineral density (BMD) of the interalveolar septum and the mandibular inferior cortex region were calculated. Tooth diameter was also measured.

Results: The VBS of the interalveolar septum in the BMAL1-KO mice was significantly deeper than that in wild-type mice. The BMD in the BMAL1-KO mice was significantly lower than in the wild-type mice in both regions. No significant difference was observed in tooth diameter between BMAL1-KO and wild-type mice.

Conclusion: These results suggest that low BMD in the interalveolar septum accelerates bone resorption in the interalveolar septum in BMAL1KO mice.
\end{abstract}

Keywords; BMAL1, bone mineral density, circadian rhythm, periodontitis

\section{Introduction}

Periodontal disease is the one of the most common inflammatory diseases in the oral cavity, affecting $20-50 \%$ of the global population [1]. The pathology of advanced periodontal disease and chronic periodontitis is characterized by destruction of the alveolar bone and periodontal ligament by immune and inflammatory responses attributable to oral microbiota, including anaerobic bacteria such as Porphyromonas, Aggregatibacter, and Prevotella [2]. It has been recognized that in addition to bacterial factors, various host and environmental factors contribute to the pathogenesis and development of periodontal disease, such as age, sex, dry mouth, systemic disease, stress, obesity, pregnancy, smoking, medication, education, and socioeconomic status [3].

Circadian rhythms in mammals are physiological and behavioral cycles with a recurring periodicity that coordinates internal time with the external environment in a daily cycle of approximately $24 \mathrm{~h}$ [4-6]. Circadian rhythms play an important role in biological homeostasis and adaptation to

Correspondence to Dr. Kunihito Matsumoto, Department of Oral and Maxillofacial Radiology, Nihon University School of Dentistry, 1-8-13 Kanda-Surugadai, Chiyoda-ku, Tokyo 101-8310, Japan

Fax:+81-3-3219-8354 E-mail: matsumoto.kunihito@nihon-u.ac.jp

J-STAGE Advance Publication: December 14, 2020

Color figures can be viewed in the online issue at J-STAGE.

doi.org/10.2334/josnusd.20-0403

DN/JST.JSTAGE/josnusd/20-0403 fluctuating environments such as the light-dark cycle, temperature, arousal stimuli, and feeding [6]. Several studies have shown that disruption of circadian rhythms induces the development and progression of various conditions or diseases such as aging, obesity, diabetes mellitus, cardiovascular disease, malignancies, psychiatric diseases, and sleep disorders [6-8].

Circadian rhythms are regulated by coordinated action between the central circadian clock system located in the suprachiasmatic nucleus and the peripheral clock system located in almost all cells of the body [9]. The essence of molecular interaction in the mammalian circadian rhythm is the transcription-translation feedback loop of brain and muscle arnt-like protein-1 (BMAL1) / circadian locomotor output cycles kaput (CLOCK)period (PER) / cryptochrome (CRY) [6,7,9]. In BMAL1-knockout (KO) mice, CLOCK mutant mice, and PER- and CRY-deficient mice, significant changes have been detected in the circadian behavioral rhythms [9]. Qiu et al. [10] reported high nocturnal locomotion and reduced sleep in BMAL1KO macaque monkeys. A previous study by Haque et al. [11] showed that BMAL1-KO mice were subject to reduced lifespan, sarcopenia, and premature aging.

It has been recognized that the circadian rhythm in mammals is also closely related to bone metabolism [12]. Reduced bone volume in BMAL1-KO and CLOCK-KO mice, and increased bone volume in PERand CRY-deficient mice have been reported [12]. However, no research showing a relationship between alveolar bone and circadian rhythm has been published. Thus, this study was conducted to radiographically evaluate morphological characteristics of the interalveolar septum in circadian rhythm deficient BMAL1-KO mice.

\section{Materials and Methods}

The procedure for the experimental animals was approved by the Animal Experimentation Committee of Nihon University School of Pharmacy (AP15P024)

\section{Experimental animals}

Ten female C57/BL strain mice (5 BMAL1-KO mice and 5 wild-type mice as controls), which were bred in Nihon University School of Pharmacy, were included in the study. The mice were housed with five animals per cage in a facility maintained at $23 \pm 1^{\circ} \mathrm{C}$ with $50 \% \pm 10 \%$ relative humidity on a 12-h light / 12-h dark cycle with normal pellet feeding. The mice were sacrificed at 36 weeks at Nihon University School of Pharmacy by carbon dioxide. Dissected heads of the mice fixed in $10 \%$ formalin neutral buffer solution were transferred to the Department of Oral and Maxillofacial Radiology Nihon University School of Dentistry. There was no information on body weight.

\section{Micro-computed tomography (micro-CT)}

Micro-CT examination was performed using the $\mathrm{R}$ m $\mathrm{CT}$ system (Rigaku, Tokyo, Japan) for all subjects. Imaging conditions were as follows: tube voltage $90 \mathrm{kV}$, tube current $200 \mu \mathrm{A}$, exposure time $2 \mathrm{~m}$, voxel size $20 \times 20 \times 20 \mu \mathrm{m}^{3}$, matrix size $480 \times 480 \times 480$. The center of $\mathrm{x}, \mathrm{y}$ and $\mathrm{z}$ axis of the FOV was set at the center of the mandible at molar region, 

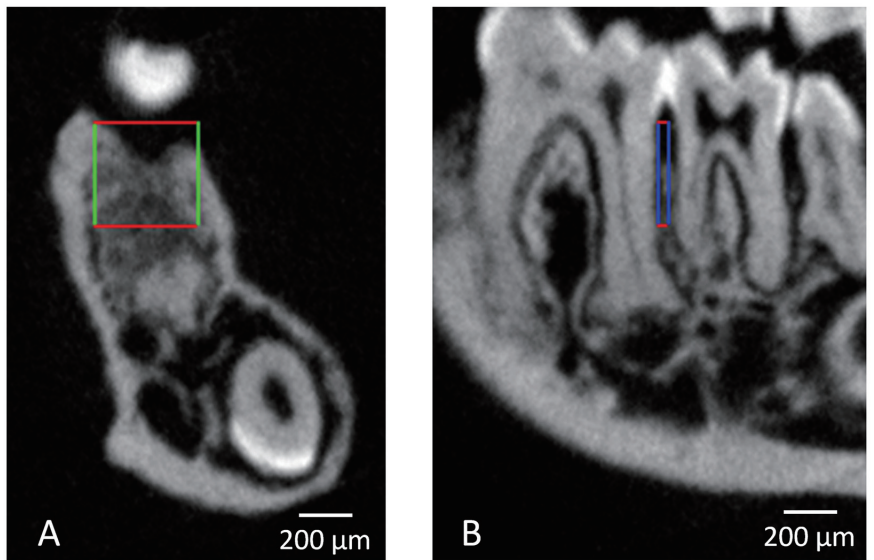

Fig. 1 Location of the volume of interest (VOI) in the interalveolar septum between the first and second molars. A: Cross-sectional slice image. B: Parasagittal slice image. The VOI was set in accordance with the axis of the interalveolar ridge between the first and second molars on the sagittal image parallel to the dental arch (A). The buccolingual center of the VOI was adjusted to the buccolingual center of the first molar on the cross-sectional image vertical to the dental arch. The superior edge of the VOI was also set at the lowest point of the cementoenamel junction at the distal interproximal surface of the first molar.
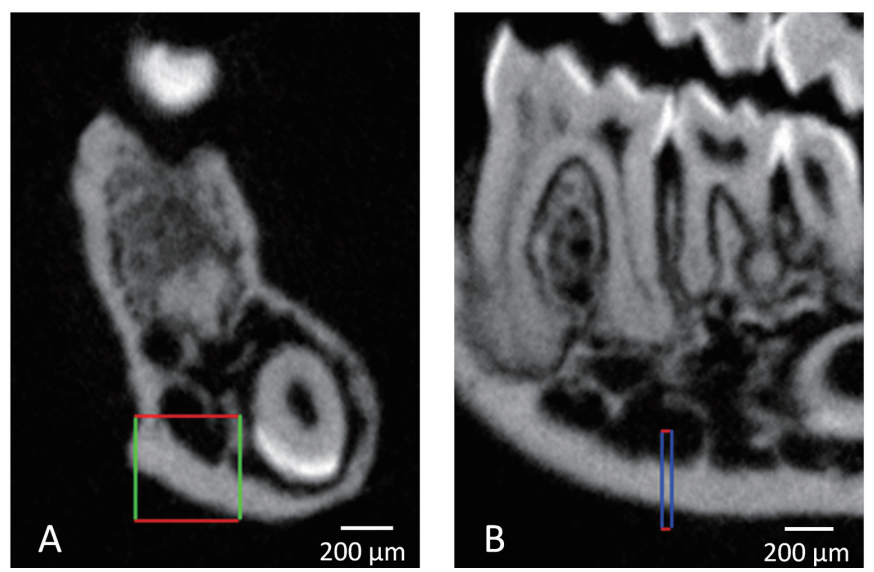

Fig. 3 Location of the volume of interest (VOI) in the inferior mandibular cortex. A: Crosssectional slice image. B: Parasagittal slice image. The VOI was set at the inferior border of the mandible corresponding to the interalveolar septum between the first and second mandibular molars. The lower and medial edges were at the lower and medial margins of the mandible, respectively.

occlusal plane, and anterior border of mandibular first molar, respectively. The imaging datasets were stored in an image server before image evaluation.

\section{Image processing and measurements}

The cross-sectional and transverse images to the mandibular molars were reconstructed with i-view software (J. Morita MFG, Kyoto, Japan). This study employed original CT images analyzer software (3by4viewver Ver. 2, Kitasenju Radist Shika, Tokyo, Japan) and virtual bone sounding analyzer software (Depth analyzer Ver. 1, Nihon University School of Dentistry, Tokyo, Japan) to measure the four items of both sides of the mandible described below.

\section{Virtual bone sounding (VBS) value}

A cuboid volume of interest (VOI), which was $400 \times 400 \times 40 \mu \mathrm{m}$ (20 pixels in both buccolingual and craniocaudal dimensions, and 2 voxels in the mesiodistal dimension), was set in the interalveolar septum between the first and second mandibular molars on the cross-sectional image of the mandible (Fig. 1). The buccolingual center of the VOI was positioned against the buccolingual center of the mandibular first molar. The superior edge of the VOI was also set at the lowest point of the cementoenamel junction (CEJ) on the distal interproximal surface of the first molar. A calibration phantom (Ratoc phantom hydroxyapatite, Ratoc System Engineering, Tokyo, Japan) was employed to measure an accurate mineral-equivalent value in milligrams per cubic centimeter of calcium
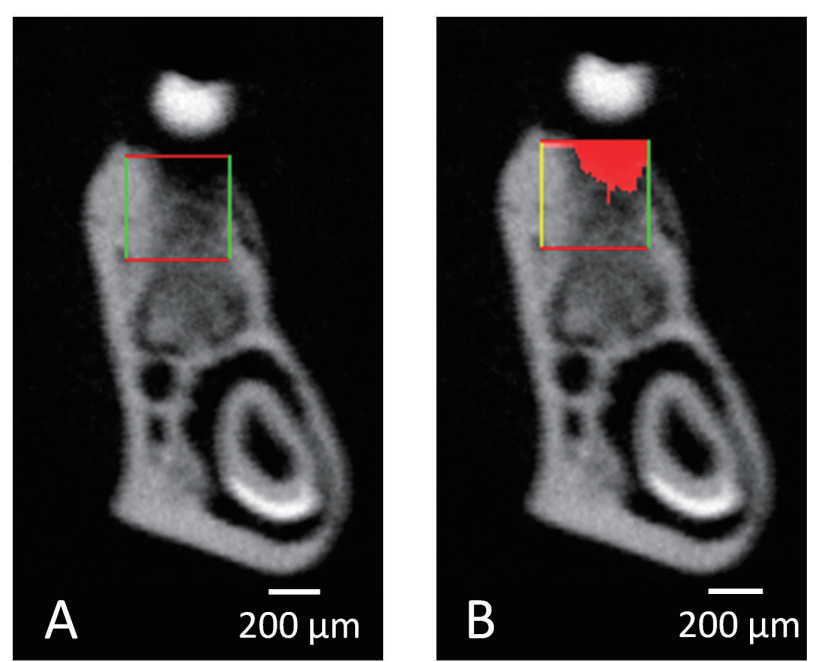

Fig. 2 Extraction of interalveolar septum in the volume of interest (VOI). A: The VOI set on the image. B: The red area in the VOI indicates the area except for bone in the interalveolar septum $\left(<300 \mathrm{mg} \mathrm{HA} / \mathrm{cm}^{3}\right)$

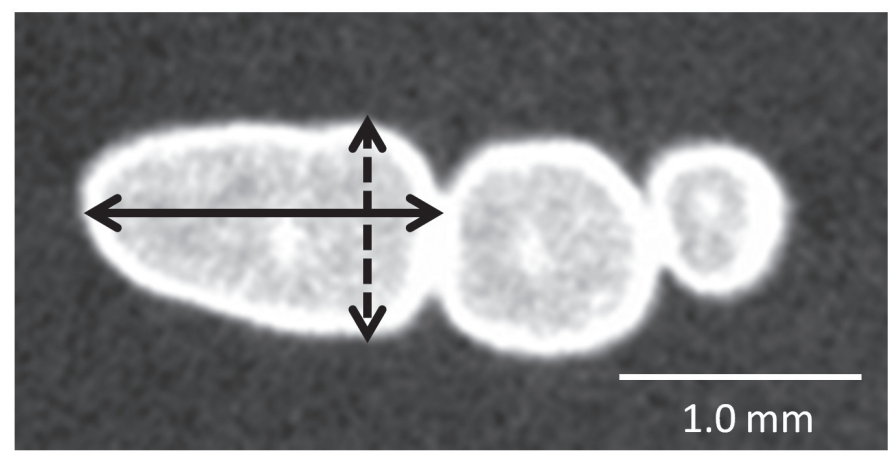

Fig. 4 Measurements of tooth diameter. Maximum mesiodistal (solid double-headed arrow) and buccolingual (dotted double-headed arrow) diameters of the crowns of the mandibular second molars were measured on horizontal slice images.

hydroxyapatite as $200,300,400,500,600,700$ and $800 \mathrm{mg} \mathrm{HA} / \mathrm{cm}^{3}$. The threshold of bone was set at over $300 \mathrm{mg} \mathrm{HA} / \mathrm{cm}^{3}$. In Fig. 2, the red area shows the area of defect in the interalveolar septum, which was the volume from the CEJ to boundary line in the interalveolar septum. The VBS value was defined as the mean depth from the CEJ to the alveolar ridge in the $\mathrm{VOI}$ and was calculated using the following formula: $\mathrm{VBS}=$ volume from the CEJ to boundary line in the interalveolar septum / (buccolingual size of the VOI $\times$ mesiodistal size of the VOI)

Bone mineral density (BMD) of the interalveolar septum

The VOI was set in the interalveolar septum as well as measuring VBS. Mean BMD was automatically calculated using a bone density curve which was initially made using a calibration phantom to measure an accurate mineral-equivalent value. BMD was defined as bone mineral density in the bone structure $\left(>300 \mathrm{mg} \mathrm{HA} / \mathrm{cm}^{3}\right)$ in the VOI.

\section{$B M D$ of the inferior cortex of the mandible}

The VOI was set at the inferior border of the mandible corresponding to the location of the interalveolar septum between the first and second mandibular molars. The lower and medial edges were at the lower and medial margins of the mandible, respectively (Fig. 3). Other conditions were similar to those used to measure the BMD of the interalveolar septum.

\section{Tooth diameter of the mandibular second molars}

The maximum mesiodistal and buccolingual diameters of the crowns of the mandibular second molars were measured on horizontal slice images that were parallel to the occlusal plane of the mandibular molars (Fig. 4).

All measurements were performed by one periodontist. The collected measurements were used to compare BMAL1-KO and wild-type mice. 
Table 1 Comparison of virtual bone sounding value and bone mineral density between BMAL1-KO and wild-type mice

\begin{tabular}{|c|c|c|c|c|c|c|c|}
\hline & \multicolumn{3}{|c|}{ BMAL1-KO mice } & \multicolumn{3}{|c|}{ Wild-type mice } & \multirow{2}{*}{$P$ value } \\
\hline & Mean & $\mathrm{SD}$ & $95 \% \mathrm{CI}$ & Mean & SD & $95 \% \mathrm{CI}$ & \\
\hline Virtual bone sounding value & $\begin{array}{l}316.2 \\
(\mu \mathrm{m})\end{array}$ & 64.9 & $269.8-362.6$ & $\begin{array}{l}253.0 \\
(\mu \mathrm{m})\end{array}$ & 65.8 & 205.9-300.0 & $<0.05$ \\
\hline $\begin{array}{l}\text { Bone mineral density } \\
\text { (interalveolar septum) }\end{array}$ & $\begin{array}{c}520.4 \\
\left(\mathrm{mg} \mathrm{HA} / \mathrm{cm}^{3}\right)\end{array}$ & 45.5 & $487.9-552.9$ & $\begin{array}{c}665.1 \\
\left(\mathrm{mg} \mathrm{HA} / \mathrm{cm}^{3}\right)\end{array}$ & 68.3 & $616.2-714.0$ & $<0.01$ \\
\hline $\begin{array}{l}\text { Bone mineral density } \\
\text { (mandibular inferior cortex) }\end{array}$ & $\begin{array}{c}716.4 \\
\left(\mathrm{mg} \mathrm{HA} / \mathrm{cm}^{3}\right)\end{array}$ & 27.7 & $696.6-736.2$ & $\begin{array}{c}825.8 \\
\left(\mathrm{mg} \mathrm{HA} / \mathrm{cm}^{3}\right)\end{array}$ & 18.2 & $812.8-838.8$ & $<0.01$ \\
\hline
\end{tabular}

BMAL1-KO, brain and muscle arnt-like protein-1 knock out; SD, standard deviation; CI, confidence interval; HA, hydroxyapatite
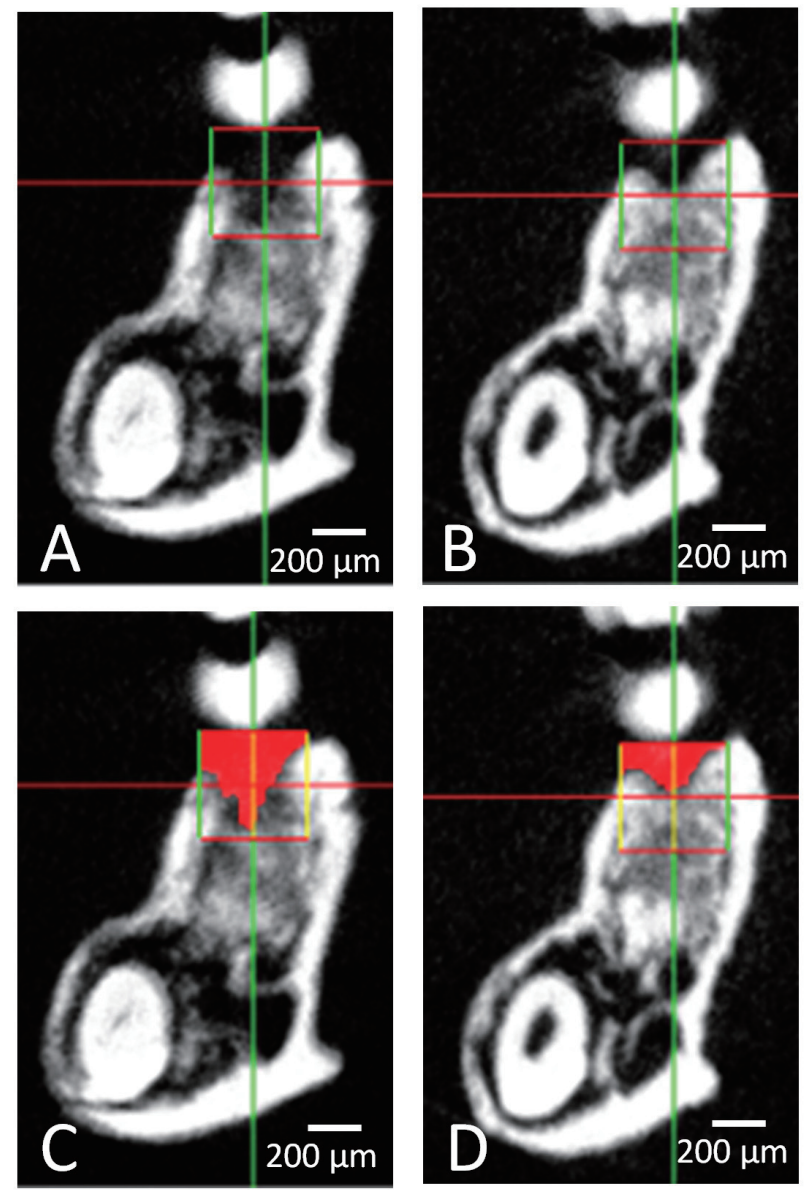

Fig. 5 Representative cross-sectional images in calculating virtual bone sounding value. Brain and muscle arnt-like protein-1 knockout (BMAL1-KO) mice showed more severe bone loss in the interalveolar septum than in wild-type mice. Volume of interest (VOI) was set in the alveolar septum of the BMAL1-KO (A) and wild-type (B) mice. Red area indicates volume from the CEJ to boundary line of the interalveolar septum in the VOI in the BMAL1-KO (C) and wild-type (D) mice.

\section{Statistical analysis}

Shapiro-Wilk and Levene's tests were performed to confirm the normality and homoscedasticity of the measurements, respectively. In measurements with normal distribution and homoscedasticity, Student's $t$-test was used for the comparison between BMAL1-KO and wild-type mice. Mann-Whitney's $U$-test was employed for analysis of abnormally distributed data. All statistical analyses were performed using SPSS Statistics for Windows version 25.0 (IBM Corp., Armonk, NY, USA). The statistical significance level was set at a $P$ value of $<0.05$.

\section{Results}

\section{VBS value}

The mean VBS value in the BMAL1-KO mice (mean $316.2 \mu \mathrm{m}$ ) was significantly higher than that in the wild-type mice (mean $253.0 \mu \mathrm{m} ; P<0.05$, Table 1 and Fig. 5).

\section{BMD of the interalveolar septum}

The mean BMD of the interalveolar septum in the BMAL1-KO mice (mean $520.4 \mathrm{mg} \mathrm{HA} / \mathrm{cm}^{3}$ ) was significantly lower than that in the wild-type mice (mean $665.1 \mathrm{mg} \mathrm{HA} / \mathrm{cm}^{3} ; P<0.01$, Table 1).

\section{BMD of the inferior cortical margin of the mandible}

The mean BMD of the inferior cortical margin of the mandible in the BMAL1-KO mice (mean $716.4 \mathrm{mg} \mathrm{HA} / \mathrm{cm}^{3}$ ) was significantly lower than that in the wild-type mice (mean $825.8 \mathrm{mg} \mathrm{HA} / \mathrm{cm}^{3} ; P<0.01$, Table 1).

\section{Tooth diameter of the mandibular second molars}

Means of the mesiodistal diameter of the mandibular second molars in BMAL1-KO and wild-type mice were $1553.0 \mu \mathrm{m}$ and $1540.0 \mu \mathrm{m}$, respectively.

Medians of the mesiodistal diameter of the mandibular second molars in BMAL1-KO and wild-type mice were $935.0 \mu \mathrm{m}$ and $920.0 \mu \mathrm{m}$, respectively.

No significant difference was detected in either the mesiodistal or buccolingual diameter of the second molars in the BMAL1-KO mice compared with wild-type mice $(P>0.05$, Table 2$)$.

\section{Discussion}

In this study, BMD in the interalveolar septum and inferior cortex of the mandible were lower in BMAL1-KO mice than in wild-type mice. Similar results were identified in the BMD of the tibia and femur in BMAL1-KO animals [12]. Additionally, BMAL1-KO animals have been shown to have low bone mass and short bone formation in long bones and the mandibular condyle $[12,13]$. A deficiency of BMAL1 promotes osteoclast differentiation and suppresses differentiation of osteoblasts and chondrocytes [14,15], finally resulting in reduced bone mass and BMD. Thus, the decrease in BMD in the interalveolar septum and the inferior cortex of the mandible in BMAL1-KO mice may be one phenotype of BMAL1 deficiency. Other clock gene $\mathrm{KO}$ animals have also exhibited bone metabolism abnormalities such as increased or decreased bone mass [16]. Therefore, circadian function has attracted considerable attention in the field of bone research. The present study used 36-week-old mice. In general, mice as old as 48 weeks are considered to be mature adults equivalent to 42-year-old humans (Life span as a biomarker [https://www.jax.org/research-and-faculty/researchlabs/the-harrison-lab/gerontology/life-span-as-a-biomarker] accessed on 30 June 2020). Thus, 36 -week-old mice can be considered to be in early middle-age and do not normally show abnormal decreases in BMD. In the BMAL1-KO mice, normal growth is observed until about 11 weeks, and then pathological changes in some biological functions, such as activity levels and body weight, gradually develop [17]. Zhou et al. [13] evaluated the morphology and bone volume of the mandibular condyle in BMAL1$\mathrm{KO}$ mice and showed that no significant hypoplasia or low bone volume was evident until at least 8 weeks. Thus, low BMD in the BMAL1-KO mice in the present study might not be a pathological condition, but may be a developmental or aging change in the interalveolar septum.

This study demonstrated that bone resorption in the interalveolar septum was more severe in BMAL1-KO mice than in wild-type mice, possibly because of low BMD in the interalveolar septum. It has been reported that decreased BMD is correlated with the severity of periodontal disease in postmenopausal women $[18,19]$. Night-shift working and rotating-shift working have been identified as risk factors for osteoporosis and osteoporotic fracture as a result of reduced BMD [20,21]. Another study revealed a relationship between shift work and periodontitis in middle-aged adults [22]. Thus, systemic decreases in BMD are associated with the severity of periodontal bone resorption as shown in the results of this study. 
Table 2 Comparison of the tooth diameters of mandibular second molars between BMAL1-KO and wild-type mice

\begin{tabular}{lccccc}
\hline & \multicolumn{3}{c}{ Mesiodistal diameter } & \multicolumn{2}{c}{ Buccolingual diameter } \\
\cline { 2 - 5 } & $\begin{array}{c}\text { Mean } \\
(\mu \mathrm{m})\end{array}$ & $\mathrm{SD}$ & $95 \% \mathrm{CI}$ & $\begin{array}{c}\text { Median } \\
(\mu \mathrm{m})\end{array}$ & $\mathrm{IQR}$ \\
\hline BMAL1-KO & 1553.0 & 45.0 & $1520.8-1585.2$ & 935.0 & 52.5 \\
Wild & 1540.0 & 56.1 & $1470.3-1609.7$ & 920.0 & 27.5 \\
\hline BMAL1-KO, brain and muscle arnt-like protein-1 knockout; SD, standard deviation; CI, confidence interval; IQR, interquartile range. No significant difference was
\end{tabular}

BMAL1-KO, brain and muscle arnt-like protein-1 knockout; SD, standard deviation; CI, confidence interval; IQR, interquartile range. No significant difference was
detected in either the mesiodistal or buccolingual diameter of the second molars in the BMAL1-KO mice compared with wild-type mice. Buccolingual diameter showed abnormal distribution and was analyzed with non-parametric test.

Reduced salivary flow rate and salivary contents increase the risk of periodontal disease $[23,24]$. Circadian rhythm is also associated with salivary function [25]. Some researchers have established that IgA secretion in saliva is associated with sleep and circadian rhythm [26,27]. Thus, deficiencies in the circadian rhythm may be a risk factor for periodontal disease and other oral diseases.

Aging is significantly associated with the increased morbidity of periodontal disease [28]. BMAL1-KO mice have been shown to experience early aging as seen in the increase of reactive oxygen species (ROS) in whole body tissue [29]. ROS are also strongly implicated in the pathogenesis of periodontal inflammation and alveolar bone resorption in periodontitis [30,31]. In the present study, the reason for severe alveolar bone resorption in BMAL1-KO mice was unclear, but may have been due to multiple factors related to the abnormality of the bone phenotype, the low BMD regulated by circadian rhythm disturbance, salivary dysfunction, earlier aging and ROS.

There was no significant difference in tooth diameter between the two groups in the study. BMAL1 deficiency does not regulate enamel morphology because enamel formation is completed just after birth. However, the relationship between amelogenesis and circadian rhythm has been demonstrated in in vitro studies [32,33]. Simmer et al. [32] showed that circadian oscillations are involved in the formation of cross-striations and the striae of Retzius in enamel by regulating the secretion of enamel proteins. Therefore, the degree of mineralization may be decreased in BMAL1-KO mice. However, no studies have revealed tooth size modulation in BMAL1-KO animals or circadian rhythm deficient models.

This study has limitations. First, it was a cross-sectional study and did not evaluate the time course of bone formation, resorption, growth, or aging. Second, the study employed only radiographic evaluation. Therefore, it was not possible to clarify the mechanism involved in the acceleration of bone loss in the interalveolar septum by each effect of low BMD, salivary dysfunction, earlier aging, and ROS regulated by circadian rhythm disturbance. Future research will include time-independent pathophysiological and genetic evaluation of alveolar bone changes.

In conclusion, this study revealed that 36-week-old BMAL1-KO mice exhibited lower BMD in the interalveolar septum and the inferior cortical margin of the mandible, and more significant bone loss in the interalveolar septum, when compared with wild-type mice. These results suggest that low BMD in the interalveolar septum accelerates bone resorption in the interalveolar septum in BMAL1-KO mice.

\section{Acknowledgments}

The authors would like to thank Dr. Yukiko Kai and Dr. Shoji Kawashima for their support in image processing. This work was supported by the Sato Fund and a grant from the Dental Research Center of Nihon University School of Dentistry. We thank Helen Jeays, BDSc AE, from Edanz Group (https://en-author-services.edanzgroup.com/) for editing a draft of this manuscript.

\section{Conflict of interest}

The authors have no conflict of interest to declare in association with this study.

\section{References}

1. Nazir MA (2017) Prevalence of periodontal disease, its association with systemic diseases and prevention. Int J Health Sci (Qassim) 11, 72-80.

2. Hasan A, Palmer RM (2014) A clinical guide to periodontology: pathology of periodontal disease. Br Dent J 216, 457-461.
3. Al-Mubarak S, Ciancio S, Baskaradoss JK (2014) Epidemiology and diagnosis of periodontal diseases: recent advances and emerging trends. Int J Dent 2014, 953646.

4. Golombek DA, Rosenstein RE (2010) Physiology of circadian entrainment. Physiol Rev 90, 1063-1102.

5. Zee PC, Attarian H, Videnovic A (2013) Circadian rhythm abnormalities. Continuum (Minneap Minn) 19, 132-147.

6. Xie Y, Tang Q, Chen G, Xie M, Yu S, Zhao J et al. (2019) New insights into the circadian rhythm and its related diseases. Front Physiol 10, 682.

7. Karatsoreos IN (2014) Links between circadian rhythms and psychiatric disease. Front Behav Neurosci 8, 162.

8. Rijo-Ferreira F, Takahashi JS (2019) Genomics of circadian rhythms in health and disease. Genome Med 11, 82.

9. Song C, Wang J, Kim B, Lu C, Zhang Z, Liu H et al. (2018) Insights into the role of circadian rhythms in bone metabolism: a promising intervention target? Biomed Res Int 2018,9156478 .

10. Qiu P, Jiang J, Liu Z, Cai Y, Huang T, Wang Y et al. (2019) BMAL1 knockout macaque monkeys display reduced sleep and psychiatric disorders. Natl Sci Rev 6, 87-100.

11. Haque SN, Booreddy SR, Welsh DK (2019) Effects of BMAL1 manipulation on the brain's master circadian clock and behavior. Yale J Biol Med 92, 251-258.

12. Samsa WE, Vasanji A, Midura RJ, Kondratov RV (2016) Deficiency of circadian clock protein BMAL1 in mice results in a low bone mass phenotype. Bone 84, 194-203.

13. Zhou X, Yu R, Long Y, Zhao J, Yu S, Tang Q et al. (2018) BMAL1 deficiency promotes skeletal mandibular hypoplasia via OPG downregulation. Cell Prolif 51, e12470.

14. Takarada T, Xu C, Ochi H, Nakazato R, Yamada D, Nakamura S et al. (2017) Bone resorption is regulated by circadian clock in osteoblasts. J Bone Miner Res 32, 872-881.

15. Wu QY, Wang J, Tong X, Chen J, Wang B, Miao ZN et al. (2019) Emerging role of circadian rhythm in bone remodeling. J Mol Med (Berl) 97, 19-24.

16. Chen G, Tang Q, Yu S, Xie Y, Sun J, Li A et al. (2020) The biological function of BMAL1 in skeleton development and disorders. Life Sci 253, 117636.

17. Hirai S, Hayashi Y, Ito M, Amemiya T, Dezawa K, Arai Y et al. (2018) Micro-CT observation of in vivo temporal change in mandibular condyle morphology in BMAL1 knockout mice. J Oral Sci 60, 473-478.

18. Tezal M, Wactawski-Wende J, Grossi SG, Ho AW, Dunford R, Genco RJ (2000) The relationship between bone mineral density and periodontitis in postmenopausal women. $\mathrm{J}$ Periodontol 71, 1492-1498.

19. Suresh S, Kumar TS, Saraswathy PK, Pani Shankar KH (2010) Periodontitis and bone mineral density among pre and post menopausal women: a comparative study. J Indian Soc mineral density among
Periodontol 14, 30-34.

20. Quevedo I, Zuniga AM (2010) Low bone mineral density in rotating-shift workers. J Clin Densitom 13, 467-469.

21. Kim BK, Choi YJ, Chung YS (2013) Other than daytime working is associated with lower bone mineral density: the Korea National Health and Nutrition Examination Survey 2009. Calcif Tissue Int 93, 495-501.

22. Han DH, Khang YH, Jung-Choi K, Lim S (2013) Association between shift work and periodontal health in a representative sample of an Asian population. Scand J Work Environ Health 39, 559-567.

23. Hirotomi T, Yoshihara A, Ogawa H, Ito K, Igarashi A, Miyazaki H (2008) A preliminary study on the relationship between stimulated saliva and periodontal conditions in community-dwelling elderly people. J Dent 34, 692-698.

24. Shimazaki Y, Fu B, Yonemoto K, Akifusa S, Shibata Y, Takeshita T et al. (2017) Stimulated salivary flow rate and oral health status. J Oral Sci 59. 55-62.

25. Zheng L, Seon YJ, McHugh J, Papagerakis S, Papagerakis P (2012) Clock genes show circadian rhythms in salivary glands. J Dent Res 91, 783-788.

26. Shirakawa T, Mitome M, Oguchi H (2004) Circadian rhythms of S-IgA and cortisol in whole saliva - compensatory mechanism of oral immune system for nocturnal fall of saliva secretion-. Pediatr Dent J 14, 115-120.

27. Wada M, Orihara K, Kamagata M, Hama K, Sasaki H, Haraguchi A et al. (2017) Circadian clock-dependent increase in salivary IgA secretion modulated by sympathetic receptor activation in mice. Sci Rep 7, 8802 .

28. Wu Y, Dong G, Xiao W, Xiao E, Miao F, Syverson A et al. (2016) Effect of aging on periodontal inflammation, microbial colonization, and disease susceptibility. J Dent Res 95 , 460-466.

29. Kondratov RV, Kondratova AA, Gorbacheva VY, Vykhovanets OV, Antoch MP (2006) Early aging and age-related pathologies in mice deficient in BMAL1, the core component of the circadian clock. Genes Dev 20, 1868-1873.

30. Dahiya P, Kamal R, Gupta R, Bhardwaj R, Chaudhary K, Kaur S (2013) Reactive oxygen species in periodontitis. J Indian Soc Periodontol 17, 411-416.

31. Saita M, Kaneko J, Sato T, Takahashi SS, Wada-Takahashi S, Kawamata, R et al. (2016) Novel antioxidative nanotherapeutics in a rat periodontitis model: reactive oxygen species scavenging by redox injectable gel suppresses alveolar bone resorption. Biomaterials 76, 292-301.

32. Simmer JP, Papagerakis P, Smith CE, Fisher DC, Rountrey AN, Zheng L et al. (2010) Regulation of dental enamel shape and hardness. J Dent Res 89, 1024-1038.

33. Zheng L, Seon YJ, Mourão MA, Schnell S, Kim D, Harada H et al. (2013) Circadian rhythms regulate amelogenesis. Bone 55, 158-165. 